\title{
Análisis de rentabilidad e impacto económico del uso de clembuterol de productores de bovino en Morelos, México
}

\section{Analysis of rentability and economic impact of the use of clenbuterol from bovine producers in Morelos, Mexico}

\author{
OMAÑA-SILVESTRE, José Miguel†' \& QUINTERO-RAMIREZ, Juan Manuel*“” \\ 1Colegio de Posgraduados, Posgrado en Economía, Campus Montecillo, Texcoco, Estado de México, México. \\ 2Cátedra CONACyT Ciudad de México, México.
}

\author{
ID $1^{\text {er }}$ Autor: José Miguel, Omaña-Silvestre / ORC ID: 0000-0002-5356-549X, CVU CONACYT ID: 59890 \\ "ID 1 ${ }^{\mathrm{er}}$ Coautor: Juan Manuel, Quintero-Ramírez / ORC ID: 0000-0002-1040-2690, CVU CONACYT ID: 292056
}

DOI: $10.35429 / \mathrm{JED} \cdot 2020.23 .7 .44 .56$

Recibido: Abril 10, 2020; Aceptado: Junio 30, 2020

\begin{abstract}
Resumen
En la presente investigación, la evaluación realizada con diferentes métodos, sirvió para determinar el nivel de producción y la competitividad económica de los proyectos ganaderos. La evaluación del proyecto tiene por objetivo identificar las ventajas y desventajas asociadas a la inversión, por lo que el método de análisis implementado es útil para adoptar decisiones racionales ante diferentes alternativas, sobre todo cuando un programa de desarrollo se encuentra involucrado en el proyecto, como es el caso del Programa "Proveedor Confiable" en la ganadería. Los métodos utilizados que sirvieron para aportar información sobre la generación de ingresos y el nivel de competitividad de las empresas, es el análisis y evaluación financiera. Se considera únicamente la vertiente monetaria del proyecto, con el objetivo de determinar su rentabilidad en términos de flujo de dinero. La investigación parte del estudio del Programa "Proveedor Confiable", que se enfocó a la erradicación del uso del Clembuterol en la alimentación animal, dando a conocer a los productores de carne la importancia de desarrollar procedimientos que reduzcan el potencial de contaminantes en los alimentos, que pueden dañar el producto final, y que generen un problema de salud en los consumidores.
\end{abstract}

\begin{abstract}
In the present investigation, the evaluation carried out with different methods served to determine the level of production and the economic competitiveness of livestock projects. The evaluation of the project aims to identify the advantages and disadvantages associated with the investment, so the implemented analysis method is useful for making rational decisions regarding different alternatives, especially when a development program is involved in the project, such as This is the case of the "Reliable Supplier" Program in livestock farming. Methods used that served to provide more accurate information on the generation of income and the level of competitiveness of companies, is the financial analysis and evaluation. Only the monetary aspect of the project is considered, with the objective of determining its profitability in terms of money flow. The research starts from the study of the "Reliable Supplier" Program, which focuses on the eradication of the use of Clenbuterol in animal feed, making meat producers aware of the importance of developing procedures that reduce the potential of contaminants in food, that can damage the final product, and that generate a health problem for consumers.
\end{abstract}

Profitability, Financial evaluation, Clenbuterol

Citación: OMAÑA-SILVESTRE, José Miguel \& QUINTERO-RAMIREZ, Juan Manuel. Análisis de rentabilidad e impacto económico del uso de clembuterol de productores de bovino en Morelos, México. Revista de Desarrollo Económico. 2020. 7 23:44-56.

\footnotetext{
* Correspondencia del Autor (Email: quintauro82@gmail.com)

$\dagger$ Investigador contribuido como primer autor.
} 


\section{Introducción}

En México uno de los sectores productivos en mayor desventaja y del que dependen millones de familias para sobrevivir es el sector agropecuario, desafortunadamente algunos elementos como la pobreza, el acceso a los recursos, las características de los mercados y la falta de oportunidades son solo algunos de los factores que han deteriorado el desarrollo del campo y de las actividades pecuarias de los mexicanos.

La ganadería bovina es una de las principales actividades del sector agropecuario debido a la variedad de productos que se pueden obtener de ésta (como la carne y la leche siendo las más importantes) y por su amplia relación que tiene con la agricultura para lograr un sistema de producción eficiente.

Aunado a esto, el incremento en los costos de producción, la falta de asesoría técnica, el desconocimiento de técnicas que agilicen el sistema de producción y la falta de tecnología, han hecho del sector agropecuario uno de los sectores más vulnerables en la economía del país.

Por otra parte, la evolución de la producción de carne de res durante la última década, es el resultado de la compleja interacción entre las diferentes ramas de la ganadería y la agricultura, esto con la finalidad de obtener insumos suficientes acordes a las necesidades de los animales, que puedan ser utilizados para producir ganado para abasto y productos cárnicos de alta calidad que cubran las exigencias de los consumidores (Cavallotti, et al., 2006).

Específicamente una de las actividades primarias que se ha visto afectada por la demanda del mercado, el alza en el precio de los insumos y el uso de productos ilícitos como el Clorhidrato de Clembuterol en la producción es la carne de ganado bovino en confinamiento; problemática sanitaria derivada del uso de esta sustancia como promotor de crecimiento en la alimentación animal. Estos problemas se han presentado tanto en los animales dentro de la unidad de producción como en los consumidores finales. Desde la década de los noventa, el Clembuterol ha sido causa de intoxicación en la población que consume carne y vísceras, principalmente hígado de res.
Sin embargo, el problema no solo radica en el hecho de que los productores implementan un sistema convencional de malas prácticas pecuarias y altas cantidades de anabólicos y fármacos que hacen imposible la certificación Tipo Inspección Federal (TIF) del producto (que es quien certifica la inocuidad de los productos cárnicos en México), también las autoridades han sido culpables al no establecer una regulación eficaz para esta situación. Estudios aplicados al sistema de producción de carne de bovino afirman que México es uno de los principales países en el mundo con potencial para integrarse al mercado internacional debido a sus condiciones climáticas y al control de enfermedades como la encefalopatía y la fiebre aftosa (Guerrero, et al. 2009).

En 2011 el Servicio Nacional de Sanidad, Inocuidad y Calidad Agroalimentaria (SENASICA) implementó el Programa Proveedor Confiable" (SAGARPA, 2011) a nivel Federal el cual consiste en la aplicación de buenas prácticas pecuarias y el no uso del Clorhidrato de Clembuterol en la alimentación animal, con la finalidad de obtener carne que no ocasione daños a la salud pública, cabe señalar que las buenas prácticas pecuarias son todas aquellas herramientas útiles para reducir riesgos de contaminación en unidades de producción primaria; brindando al consumidor un producto inocuo que no cause daño a su salud humana.

El objetivo de la investigación parte del estudio del mencionado Programa, que se enfoca a la erradicación del uso del Clembuterol en la alimentación animal, dando a conocer a los productores de carne la importancia de desarrollar procedimientos que reduzcan el potencial de contaminantes en los alimentos, que pueden dañar el producto final, y que generen un problema de salud en los consumidores.

Se parte de la hipótesis de que el Programa Proveedor Confiable a través de la implementación de las buenas prácticas pecuarias permite la obtención de una producción de carne de mejor calidad, libre del uso de Clembuterol, lo cual compensa el incremento en los costos de producción en comparación con la carne obtenida bajo un esquema tradicional. La compensación se obtiene al vender un producto sano y de calidad el cual es más valorado en el mercado. 
En el estado de Morelos, el sector primario se compone de todas aquellas actividades agrícolas, ganaderas, de aprovechamiento forestal, pesca y caza, estas actividades tienen una aportación al PIB estatal que oscila alrededor del 3\%, aunque cabe destacar, que las actividades del sector primario no son las de mayor empuje en el estado, pero si juegan un papel esencial dentro de la economía de la población.

Para la presente investigación se analizó la rentabilidad del sistema de producción utilizado por los ganaderos de una Sociedad de Producción Rural de Responsabilidad Limitada de Ganaderos, que ha establecido 36 unidades de producción (U.P.) en diferentes municipios del estado de Morelos; con el fin de demostrar que tan rentable es producir bajo el esquema del Programa "Proveedor Confiable" y conocer los beneficios que pueden alcanzar los productores si se apegan a la ley y a la normatividad establecida en México para la producción y comercialización de alimentos inocuos.

La importancia de la investigación se enfoca en el análisis de rentabilidad de un sistema de producción ganadero, basado en las normas del Programa Gubernamental, que tiene como objetivo la producción de carne de ganado bovino bajo un esquema poco ordinario que promete el desarrollo de la actividad y una mayor generación de ingresos para los productores.

Se pretende demostrar que la engorda de ganado bovino cuando es llevada a cabo mediante las normas de un programa establecido, puede resultar en la obtención de un producto de calidad, libre de contaminantes, que sea más competitivo en el mercado y que permita reducir costos de producción, aumentar el rendimiento, y cubrir la demanda de mercados más exigentes.

\section{Metodología}

La evaluación realizada del Programa "Proveedor Confiable" y el impacto que ha tenido sobre las unidades de producción, fue llevada a cabo mediante el diseño de un cuestionario dirigido al representante oficial de la delegación estatal de la SAGARPA, y a los productores que actualmente participan en él.
La investigación se llevó a cabo mediante la identificación y el análisis de los distintos factores económicos que intervienen directamente en el sistema de producción de carne bovina en el Estado de Morelos.

Se realizó un diagnóstico general sobre la S.P.R. de R.L. "Bovinos del Corral al Plato", con el cual se pudo conocer y evaluar la información más relevante de los sistemas implementados por cada una de las 13 unidades de producción que actualmente se encuentran activas; de las cuales 8 unidades cuentan con constancia de proveedor confiable y 5 unidades con oficio de Hato Libre.

El estudio de impacto económico se llevó a cabo mediante un muestreo dirigido. El método de muestreo fue seleccionado debido al tamaño de la población y al acceso a la información que se presentó por cada uno los productores.

Mediante este método se realizó una clasificación de tres grupos, dónde se asignó un número de identificación que facilito el análisis de las unidades productivas.

La clasificación de los grupos se realizó con base a la comparación de la capacidad instalada y del tamaño de la unidad productiva, dicho de otro modo, esta agrupación se deriva de organizar la información obtenida en cuanto a los volúmenes de producción, ver tabla 1.

Grandes: Unidades de producción que manejan volúmenes superiores a las 80 cabezas de ganado y poseen superficies mayores a 1 hectárea de infraestructura.

Medianos: Unidades de producción que se encuentran en un rango de producción de 30 a 80 cabezas de ganado por ciclo productivo y que cuentan con más de $1000 \mathrm{~m}^{2}$, pero menos de $5000 \mathrm{~m}^{2}$ de infraestructura.

Pequeños: Unidades de producción que trabajan con volúmenes menores a 30 cabezas de ganado por ciclo productivo y con superficies menores a los $1000 \mathrm{~m}^{2}$ de infraestructura.

Una vez determinadas las muestras se procedió a realizar un estudio detallado del sistema de producción y las características físicas de la unidad productiva. 


\begin{tabular}{|c|c|c|c|c|c|}
\hline \multirow[t]{2}{*}{$\begin{array}{c}\text { Tamaño } \\
\text { U.P. }\end{array}$} & U.P. & \multicolumn{2}{|c|}{$\begin{array}{l}\text { Capacidad } \\
\text { Instalada }\end{array}$} & \multicolumn{2}{|c|}{ Capacidad Usada } \\
\hline & $\mathbf{N}^{\circ}$ & $\begin{array}{c}\mathbf{N}^{\circ} \\
\text { cabezas }\end{array}$ & $\%$ & $\begin{array}{c}\mathbf{N}^{\circ} \\
\text { Cabezas }\end{array}$ & $\%$ \\
\hline \multirow[t]{3}{*}{ Grandes } & 1 & 350 & 100 & 165 & 47.14 \\
\hline & 2 & 250 & 100 & 142 & 56.80 \\
\hline & 3 & 150 & 100 & 82 & 54.67 \\
\hline \multirow[t]{5}{*}{ Medianas } & 4 & 130 & 100 & 60 & 46.15 \\
\hline & 5 & 130 & 100 & 58 & 44.62 \\
\hline & 6 & 60 & 100 & 45 & 75.00 \\
\hline & 7 & 52 & 100 & 40 & 76.92 \\
\hline & 8 & 50 & 100 & 32 & 64.00 \\
\hline \multirow[t]{5}{*}{ Pequeñas } & 9 & 28 & 100 & 20 & 71.43 \\
\hline & 10 & 30 & 100 & 17 & 56.67 \\
\hline & 11 & 20 & 100 & 14 & 70.00 \\
\hline & 12 & 12 & 100 & 12 & 100 \\
\hline & 13 & 20 & 100 & 9 & 45.00 \\
\hline
\end{tabular}

Tabla 1 Clasificación de las muestras

Fuente: Elaboración propia con datos obtenidos del muestreo dirigido realizado a la S.P.R. de R.L.

Una vez clasificada la información se realizó un análisis cuantitativo del sistema productivo enfocado a la rentabilidad $y$ generación de ingresos. Se analizaron los conceptos más representativos que componen el análisis financiero, mismos que sirvieron para el cálculo de los indicadores de rentabilidad.

Mediante el análisis de resultados se determinó el grado de competitividad que cada una de las muestras presenta durante su ciclo de producción. Con esta información se llevó a cabo una propuesta de desarrollo con base a la aplicación del Programa "Proveedor Confiable" en el sistema de producción y como es que afecta el ingreso del productor.

\section{Indicadores Económicos}

La investigación utiliza parte del esquema de la Matriz de Análisis de Política (MAP) desarrollado por Erick Monke y Scott R. Pearson (1989) de las Universidades de Arizona y Stanford, respectivamente.

El análisis se complementa con la metodología de análisis de Ingreso y de Inversión del Instituto de Desarrollo Económico del Banco Mundial.

En la MAP el análisis de las ventajas comparativas se construye con base en los presupuestos privados y económicos y tiene la finalidad de medir los efectos de apertura comercial y la eficiencia en los sistemas de producción regionales.
Los precios privados son los precios de mercado actuales, mientras que los precios de eficiencia económica, también llamados sociales, son aquellos que reflejan valores de escasez o costos de oportunidad económica. Para esta investigación solo se utiliza la parte privada, es decir, los presupuesto privados a precios que paga o recibe el productor por la compra de insumos y la venta de su producto respectivamente.

Dos identidades de contabilidad están determinadas en la MAP. La primera mide la ganancia como la diferencia entre ingresos y costos de producción.

$$
\text { Ganacias }=\sum P_{i} Q_{i}-\left[\sum P_{j} Y_{j}+\sum P_{k} Z_{k}\right]
$$

Donde: nacional.

$P i$ : precio del producto en el mercado hectárea.

$X i$ : cantidad de toneladas producidas por

$P j$ : precio de los insumos comerciables en el mercado nacional.

$Y j$ : cantidad de insumos comerciables e indirectamente comerciables aplicados por hectárea.

$P k$ : precio de los factores internos en el mercado nacional.

$Z k$ : cantidad de factores internos aplicados por hectárea.

La segunda identidad mide los efectos de política y las distorsiones de mercado y está determinada por las diferencias entre las evaluaciones privadas y las económicas de los ingresos, costos y ganancias.

Los costos de producción incluyen insumos comerciables y factores internos. Dentro de los insumos comerciables se consideran los insumos o parte de ellos, que se pueden adquirir en los mercados, tanto nacional como internacional, por ejemplo: alimentos, medicamentos, vacunas, diésel y partes de maquinaria. 
Los insumos indirectamente comerciables incluyen insumos o parte de ellos que no son comercializados internacionalmente, como son los implementos y alguna maquinaria usada en la producción de carne.

\section{La rentabilidad privada}

La rentabilidad privada medida a través de las ganancias privadas, es decir, la diferencia entre los ingresos totales de ventas (o por unidad) y los costos de mercado de los insumos comerciables $\mathrm{y}$ factores internos. Tanto ingresos como costos se trabajan previamente, multiplicando los coeficientes técnicos por sus respectivos precios privados. El cálculo de la rentabilidad privada muestra la competitividad del sistema de producción, dadas las tecnologías actuales y los precios del producto y los factores que están imperando en ese momento en el mercado, así como las transferencias o impuestos generados por medidas de política económica.

De acuerdo con el Banco Mundial, la producción bruta de la finca se divide en dos componentes principales: El Valor agregado neto: que es la contribución de la actividad agrícola a ingreso del propio sector, y el Consumo Intermedio, que es el gasto de la actividad económica analizada en otros sectores de la economía regional. Se compone de los gastos corrientes de la finca (exceptuando salarios) y el pago por la utilización de los equipos agroindustriales de uso pecuario.

Guerra E. (1997) señala que para el estudio financiero del agronegocio el resultado del valor agregado neto puede ser determinado mediante el análisis con la siguiente expresión:

$V A G N=I-(I C+E+I I C+G D)$

Donde:

$V A G N=$ Valor agregado neto

$I=$ Ingreso total

$I C=$ Costo de Insumos comerciables

$E=$ Gasto de Electricidad

comerciables

$I I C=$ Costo Insumos indirectamente

$G D=$ Gastos diversos
La suma entre paréntesis del costo de los insumos comerciables, electricidad, insumos indirectamente comerciables y gastos diversos constituyen el Consumo Intermedio.

El Valor Agregado Neto es un concepto financiero más completo que señala la contribución de la actividad económica analizada al interior del sector.

La diferencia entre los ingresos obtenidos en la producción de carne y los costos que implica esta producción, constituyen las ganancias, o perdidas, que está generando la actividad analizada. Sin embrago, al tratar de explicar esta ganancias o pérdidas se puede establecer algunos indicadores de rentabilidad como es la Remuneración al Capital del productor la cual representa la cantidad de dinero que se gana por cada peso invertido, se calcula con la siguiente ecuación:

$R C=V A G N-(P P+P O+U A)$

Donde:

$$
\begin{aligned}
& R C=\text { Remuneración al capital } \\
& V A G N=\text { Valor agregado neto } \\
& P P=\text { Gastos Personal profesional } \\
& P O=\text { Gastos Personal operario } \\
& U A=\text { Gasto por el uso del agua }
\end{aligned}
$$

La RC es un cálculo financiero que arroja un resultado más completo sobre la cantidad de dinero que se gana al finalizar el proceso de producción.

Asimismo, al dividir la remuneración absoluta al capital entre el total de costos implicados en la producción se obtiene la remuneración relativa al capital, la cual se determina mediante la siguiente ecuación:

$$
R R C=\frac{R C}{(I C+E+I I C+P P+P O+U A+C)}
$$

Donde:

$$
\begin{aligned}
& R R C=\text { Remuneración relativa al capital } \\
& R C=\text { Remuneración al capital } \\
& I C=\text { Costo de Insumos comerciables }
\end{aligned}
$$

OMAÑA-SILVESTRE, José Miguel \& QUINTERORAMIREZ, Juan Manuel. Análisis de rentabilidad e impacto económico del uso de clembuterol de productores de bovino en Morelos, México. Revista de Desarrollo Económico. 2020 
$E=$ Gasto de Electricidad

$I I C=$ Costo Insumos ind. Comerciables

$P P=$ Gasto del Personal profesional

$P O=$ Gasto de Personal operario

$U A=$ Gasto por el uso del agua

$C=$ Créditos

\section{Análisis de competitividad o relación de costo privado}

Para conocer el nivel de competitividad económica de la empresa, Aguilar Valdés (1997) señala que la competitividad económica o Relación de Costo Privado de la empresa es igual cociente que resulta de dividir la suma de los factores internos entre el resultado del valor agregado neto, es decir:

$R C P=\frac{F I}{V A G N}$

Donde:

$R C P=$ Relación del costo privado

$F I=$ Factores internos

$V A G N=$ Valor agregado neto

Dentro de los posibles resultados una RCP mayor que la unidad o negativo, implica que:

- Al realizar la actividad el sistema productivo no permita pagar el valor de mercado de los factores internos (incluyendo una tasa de retorno normal al capital).

- La ganancia resulta negativa.

- La actividad no es redituable para el productor en función de los precios pagados y recibidos no siendo competitivo.

RCP menor que la unidad, implica que:

- Producir la actividad genera que el sistema productivo si permite pagar el valor de mercado de los factores internos (incluyendo una tasa de retorno normal al capital).
- La ganancia resulta positiva.

- La actividad es redituable para el productor en función de los precios pagados $\mathrm{y}$ recibidos.

Nota: La minimización de RCP genera la máxima ganancia.

El RCP sirve para determinar qué tan bien se emplean los recursos obtenidos durante el ciclo de producción o, dicho de otro modo, cuanto del capital generado se utiliza en el pago de los factores internos de la empresa.

\section{Resultados}

\section{Distribución de unidades de producción}

En el estado de Morelos, la distribución de las actividades agropecuarias no se encuentra establecidas en zonas definidas como puede observarse en otros estados de la república, aquí las actividades pecuarias se encuentran distribuidas en gran parte del estado siendo pequeñas unidades de producción con un número que no supera las 30 cabezas de ganado en promedio.

La ubicación geográfica puede ser un factor determinante para la generación de mayores ingresos, si bien, es sabido que las ganaderías que se encuentran más cerca del punto de comercialización han demostrado un mayor crecimiento tanto económico como productivo, también es de considerar, los logros obtenidos por aquellas unidades que se encuentran lejos del área de comercialización, y que a pesar de tener desventajas tales como la distancia entre el rastro de sacrificio y la unidad de producción, han logrado marcar un significativo avance en lo relacionado a sus ingresos y a sus productos finales.

En la Figura 1, se muestra la distribución de las 13 unidades de producción de estudio, colindantes con los estados de Puebla, Guerrero, Estado de México y Ciudad de México. 


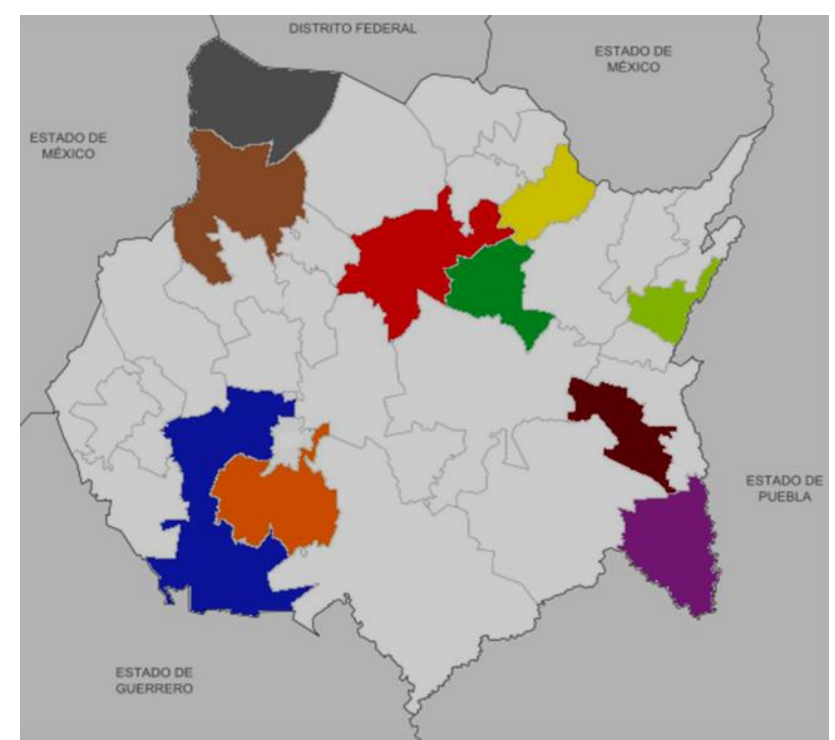

Figura 1 Distribución de las unidades de producción activas de la S.P.R. en el estado de Morelos

Fuente: Elaboración propia con datos de la S.P.R de R.L.

La distribución regional de las ganaderías abarca 10 de los 33 municipios del estado, siendo Yautepec el municipio que alberga a 4 de las unidades de producción, las cuales manejan un mayor número de cabezas de ganado y otras se encuentran situadas en los municipios de Puente de Ixtla y Jojutla.

Las ganaderías situadas en los municipios de Atlatlahucan, Cuernavaca, Huitzilac, Cuautla y Zacualpan de Amilpas, son unidades que en promedio manejan de 20 a 60 cabezas de ganado, con la importante ventaja de situarse a menor distancia del área o punto de comercialización.

En los municipios de Axochiapan y Jonacatepec únicamente se observan dos unidades de producción, en Jonacatepec la actividad productiva se enfoca a la producción de sorgo y otros granos, por tal motivo, dentro del municipio se localiza uno de los principales centros de distribución y comercialización de insumos para la S.P.R. de R.L.

\section{Unidades de producción en relación con el área de comercialización}

Factores como la distancia y los tiempos de traslado del ganado finalizado hacia el área de comercialización, son algunas de las causas por la cual se pueden derivar problemas de estrés en los animales, mermas del productos y mayores gastos de movilización.
Esta relación permite determinar que en actividades productivas como la ganadería, cuando existe una mayor distancia entre la unidad de producción y el área de comercialización, se aumenta el riesgo de contraer mayores pérdidas económicas, sin dejar de lado la teoría de que estas pérdidas pueden ser reducidas mediante la elaboración de un plan de movilidad, que contemple acciones preventivas $y$ eficientes dentro y fuera de la unidad de producción.

La Figura 2, muestra la relación de distancia que existe entre las unidades de producción y el área de comercialización y sacrificio, en él se pueden observar las rutas a seguir y los tiempos estimados que los productores tardan en llegar a este último punto.

El punto de comercialización se encuentra ubicado en el municipio de Yautepec, así mismo, el área de mercado o el área de distribución de los productos se encuentra conformada por los municipios de Cuautla, Cuernavaca, Yautepec, Tlayacapan, Yecapixtla y Tepoztlan, siendo Cuautla, Cuernavaca y Yautepec los municipios que mayor cantidad de carne demandan a la Distribuidora de Carnes.

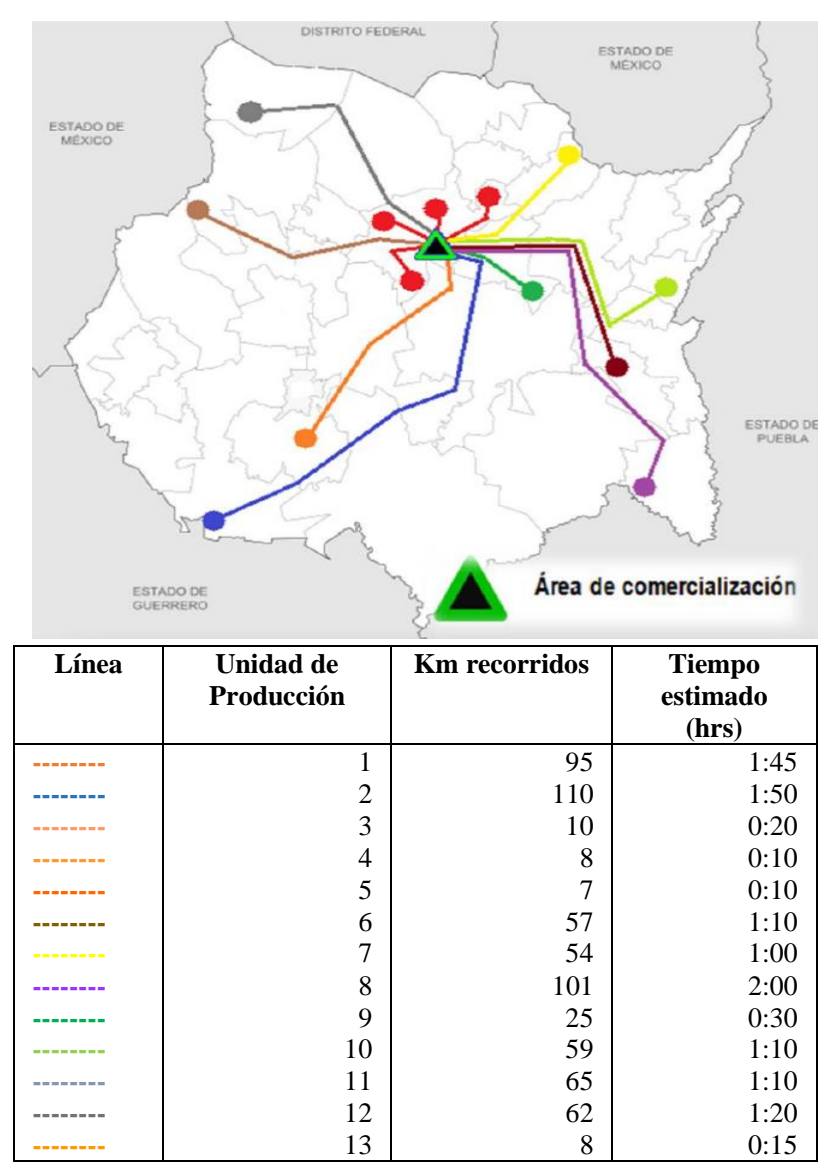

Figura 2 Relación de distancia de las unidades de producción y el área de comercialización.

Fuente: Elaboración propia con datos de la S.P.R de R.L. 
En relación con la distancia, existe una clara diferencia entre los costos establecidos por unidad de producción, puesto que las mermas y los gastos de transporte son directamente proporcional a los kilómetros recorridos.

\section{Adquisición y movilización del ganado}

Para el inicio de la engorda de ganado bovino en confinamiento comienza con la adquisición del becerro, proveniente de estados aledaños como, Oaxaca, Guerrero, Tabasco y Veracruz, donde el precio de compra es uno de los puntos claves para determinar los ingresos, ya que dicho precio se liga al peso, edad y genética de los animales que depende de las condiciones climáticas y la programación de sacrificio que cada productor tiene. Por otra parte, hay que tomar en cuenta que durante la adquisición del ganado los costos de movilización juegan un papel importante, puesto que estos son directamente proporcionales a la distancia que recorren desde su origen y hasta la unidad de producción, además de que varían de acuerdo con el medio de transporte utilizado.

La tabla 2 muestra el precio de adquisición del ganado en los estados antes mencionados es muy similar, aunque relativamente más bajo en el estado de Guerreo, sin embargo, los costos de movilización se incrementan cuando el ganado proviene de estados como Tabasco y Oaxaca, puesto que la distancia recorrida es mayor y el número de inspecciones zoosanitarias aumenta considerablemente para llegar a la unidad de producción de destino.

\begin{tabular}{|l|r|r|r|}
\hline \multicolumn{1}{|c}{$\begin{array}{c}\text { Unidad de } \\
\text { producción }\end{array}$} & Pequeñas & \multicolumn{1}{c|}{ Medianas } & Grandes \\
\hline $\begin{array}{l}\text { Origen del } \\
\text { ganado }\end{array}$ & Guerrero & Tabasco & Oaxaca \\
\hline $\mathrm{N}^{\circ}$ de cabezas & 20 & 40 & 142 \\
\hline Peso inicial kg & 310 & 380 & 270 \\
\hline Precio/kg en \$ & 37.32 & 38.31 & 38.81 \\
\hline Precio/ Cabeza \$ & 11,568 & 14,558 & 10,478 \\
\hline Precio total \$ de & 231,361 & 582,333 & $1,487,933$ \\
\hline $\begin{array}{l}\text { Tipo camión } \\
\text { transporte }\end{array}$ & 2 camiones & 2 jaulas \\
\hline $\begin{array}{l}\text { Gastos de } \\
\text { movilización \$ }\end{array}$ & 6,826 & 20,300 & 57,023 \\
\hline $\begin{array}{l}\text { Costo total del } \\
\text { ganado puesto en } \\
\text { la UP en \$ }\end{array}$ & 238,187 & 602,633 & $1,544,956$ \\
\hline
\end{tabular}

Tabla 2 Comparación en relación con el costo del ganado y su movilización. Precios reales 2019. IPP 12012=100 Fuente: Elaboración propia con datos tomados de la S.P.R. de R.L.
Durante el estudio se analizaron 4 indicadores que son esenciales para medir la rentabilidad y conocer el impacto generado sobre el ingreso de los productores. Los cuatro indicadores referidos son:

- Rentabilidad privada.

- El valor agregado y consumo intermedio.

- La remuneración al capital.

- $\quad$ Competitividad (RCP).

\section{- Rentabilidad privada}

Dentro de las U.P. estudiadas de la S.P.R., no se observa algún valor que represente pérdidas para el productor, es decir, los tres tipos de empresas son rentables, siendo las empresa más grande la que genera un mayor ingreso por el número de cabezas que tiene, lo cual se refleja en este caso en una mayor ganancia por cabeza; sin embargo, la empresa mediana es la que recibió un mayor precio por cabeza, revelando un nivel de rentabilidad promedio más alto, esto debido al peso con el cual salen al mercado los animales, de $640 \mathrm{~kg}$ en comparación a los $530 \mathrm{~kg}$ de las empresas grandes y $460 \mathrm{~kg}$ de las empresas chicas.

Es importante señalar que a pesar de que producen carne libre de Clembuterol esto no garantiza que reciban un precio diferenciado por su producto, es decir, el programa no contempla un mercado diferenciado donde puedan comercializar su producción garantizando un mejor precio por esta característica.

\begin{tabular}{|l|r|r|r|}
\hline \multicolumn{1}{|c|}{ Tamaño } & Pequeñas & Medianas & Grandes \\
\hline No. Cabezas & 20 & 40 & 142 \\
\hline Peso final & 460 & 640 & 530 \\
\hline Precio/kg & 42.79 & 43.78 & 43.78 \\
\hline Precio/cabeza & 19,683 & 28,022 & 23,206 \\
\hline Ingreso total & 393,662 & $1,120,881$ & $3,295,214$ \\
\hline Costo total & 376,394 & $1,060,900$ & $3,111,186$ \\
\hline Ganancia neta & 17,268 & 59,981 & 184,028 \\
\hline $\begin{array}{l}\text { Ganancia por } \\
\text { cabeza }\end{array}$ & 863.40 & $1,499.52$ & $1,295.97$ \\
\hline
\end{tabular}

Tabla 3 Comparación de ingresos, costos y ganancias total por unidad de producción. Precios reales 2019. IPP $2012=100$

Fuente: Elaboración propia con datos de las U.P muestra de la S.P.R. de R.L. 


\section{- Costo de producción}

El análisis comparativo muestra un panorama de los sistemas de producción implementados, uno de los factores determinantes del ingreso es la alimentación, ya que, si no se cuenta con una dieta balanceada que promueva el desarrollo de los animales a un precio razonable, existe la posibilidad de generar mayores pérdidas que ganancias.

Las instalaciones juegan un papel de suma importancia para la producción, esto se debe a que los factores climáticos tales como la lluvia y los cambios de temperatura han demostrado un retraso en la engorda de bovinos que va del $3 \%$ a más del $5 \%$ de lo normal. Por esta razón, la mínima inversión en instalaciones adecuadas y la falta de un diseño estratégico, generan pérdidas importantes que pueden ser más notorias en corrales mal diseñados, que albergan bovinos de buena genética y de mayor peso.

De igual forma, la implementación de un tratamiento adecuado contra enfermedades y parásitos es uno de los principales componentes de la producción, esto se debe a que con el paso del tiempo se descubren nuevas enfermedades en el ganado bovino que no solo pueden ser contraídas en potreros, sino también, pueden llegar a un sistema intensivo por medio del agua y la alimentación suministrada.

Cuando no se implementa un correcto tratamiento de defensa contra enfermedades, se pueden presentar casos de rezago y altos índices de mortandad, que representan la pérdida más significativa para la unidad de producción (Huerta Suarez, 2012).

De acuerdo con estas consideraciones las unidades de producción grandes son las que tienen contemplado la mayoría de estas características en la producción de carne y son estas empresas cuyos costos de alimentación, vehículos e instalaciones, así como vacunas y medicamentos representan un porcentaje importante de sus costos de producción; sin embargo, en términos de costo por cabeza, son las empresas medianas las que alcanzan un costo superior. (ver Tabla 4).

\begin{tabular}{|l|r|r|r|}
\hline \multicolumn{1}{|c}{$\begin{array}{c}\text { Tamaño } \\
\text { No. Cabezas }\end{array}$} & \multicolumn{1}{c}{$\begin{array}{c}\text { Pequeñas } \\
\text { Modianas }\end{array}$} & \multicolumn{1}{c|}{$\begin{array}{c}\text { Grandes } \\
\mathbf{1 4 2}\end{array}$} \\
\hline $\begin{array}{l}\text { A)Insumos } \\
\text { Comerciables }\end{array}$ & $\mathbf{1 0 0 , 5 2 2}$ & $\mathbf{3 1 5 , 5 6 1}$ & $\mathbf{1 , 1 4 7 , 4 5 2}$ \\
\hline Alimentación & 85,257 & 265,377 & $1,017,836$ \\
\hline $\begin{array}{l}\text { Vacunas } \\
\text { medicamentos }\end{array}$ & 1,126 & 9,768 & 24,324 \\
\hline $\begin{array}{l}\text { Detergentes } \\
\text { combustibles }\end{array}$ & 14,138 & 40,416 & 105,292 \\
\hline B)Factores Internos & $\mathbf{1 6 , 5 9 8}$ & $\mathbf{6 2 , 7 0 3}$ & $\mathbf{1 2 1 , 2 8 3}$ \\
\hline Mano de obra & 16,121 & 61,696 & 119,492 \\
\hline Agua y electricidad & 478 & 1,007 & 1,791 \\
\hline C) Ins. Ind. Comer. & $\mathbf{2 4 6 , 2 2 8}$ & $\mathbf{6 6 1 , 3 4 3}$ & $\mathbf{1 , 7 8 1 , 6 8 7}$ \\
\hline Compra de ganado & 231,361 & 582,333 & $1,487,933$ \\
\hline $\begin{array}{l}\text { Veh., instal. Maq. y } \\
\text { eq. }\end{array}$ & 14,867 & 79,011 & 293,754 \\
\hline D) Gastos Diversos & $\mathbf{1 3 , 0 4 6}$ & $\mathbf{2 1 , 2 9 2}$ & $\mathbf{6 0 , 7 6 5}$ \\
\hline $\begin{array}{l}\text { Costo total (A + B + } \\
\text { C + D) }\end{array}$ & $\mathbf{3 7 6 , 3 9 4}$ & $\mathbf{1 , 0 6 0 , 9 0 0}$ & $\mathbf{3 , 1 1 1 , 1 8 6}$ \\
\hline Costo por cabeza & $18,819.68$ & $6,522.50$ & $21,909.76$ \\
\hline
\end{tabular}

Tabla 4 Costos de producción por tipo de Unidad de producción. Precios reales 2019. IPP 2012 $=100$ Fuente: Elaboración propia con datos de las U.P muestra de la S.P.R. de R.L.

\section{- Valor agregado y consumo intermedio}

De acuerdo con la Tabla 5, todas las unidades productivas, al igual que los ingresos y las ganancias, generan un valor agregado positivo, siendo las grandes las empresas que más contribuyen en la generación del valor dentro del sector pecuario. Los valores generados van de los $\$ 33,568$ hasta los $\$ 303,718$. Al comparar estos valores generado con respecto a ingreso obtenido se observa que nuevamente son las empresas medianas las que su valor agregado representa una proporción mayor con respecto al ingreso generado, del $11 \%$, esto significa que, por cada peso generado de ingreso, el $11 \%$ corresponde al valor agregado que la actividad genera.

\begin{tabular}{|l|r|r|r|}
\hline \multicolumn{1}{|c}{ Tamaño } & \multicolumn{1}{c|}{ Pequeñas } & \multicolumn{1}{c|}{ Medianas } & \multicolumn{1}{c|}{ Grandes } \\
\hline (1) Ingreso total & 393,662 & $1,120,881$ & $3,295,214$ \\
\hline $\begin{array}{l}\text { (2) Insumos } \\
\text { comerciables }\end{array}$ & 100,522 & 315,561 & $1,147,452$ \\
\hline (3) Electricidad ind. & 246,228 & 661,343 & $1,781,687$ \\
\hline $\begin{array}{l}\text { (4) Ins. indes } \\
\text { comerciables }\end{array}$ & 13,046 & 21,292 & 60,765 \\
\hline $\begin{array}{l}\text { (5) Gastos diversos } \\
\text { neto Valor agregado }\end{array}$ & 33,568 & 122,180 & 303,718 \\
\hline $\begin{array}{l}\text { \% respecto al ingreso } \\
\text { generado }\end{array}$ & $8.53 \%$ & $10.90 \%$ & $9.22 \%$ \\
\hline $\begin{array}{l}\text { (7) Intermedio } \\
\text { (2+3+4+5) }\end{array}$ & 360,094 & 998,700 & $2,991,495$ \\
\hline $\begin{array}{l}\text { \% respecto al ingreso } \\
\text { generado }\end{array}$ & $91.47 \%$ & $89.10 \%$ & $90.78 \%$ \\
\hline
\end{tabular}

Tabla 5 Valor Agregado y Consumo Intermedio por tipo de Unidad de producción. Precios reales 2019. IPP 2012=100 Fuente: Elaboración propia con datos de las U.P muestra de la S.P.R. de R.L

OMAÑA-SILVESTRE, José Miguel \& QUINTERORAMIREZ, Juan Manuel. Análisis de rentabilidad e impacto económico del uso de clembuterol de productores de bovino en Morelos, México. Revista de Desarrollo Económico. 2020 
Por otro lado, la derrama económica que cada unidad productiva hace al interior del propio sector, es decir, el consumo intermedio, son las empresas grandes las que contribuyen en mayor medida al sector por la dimensión de estas. Analizando en términos relativos son las empresas pequeñas las que hacen una derrama económica del $91.5 \%$ del ingreso que generan.

\section{- Remuneración al Capital del Productor}

El cálculo de la Remuneración al capital, tanto en términos absolutos como relativos resulta de dividir las ganancias obtenidas entre el costo total de cada una de las unidades de producción. Esta remuneración sería un acercamiento a la relación beneficio/costo si este estuviera una tasa de descuento.

La interpretación indica que son las unidades de producción grandes las que generan una mayor remuneración al capital que se invierte para la producción de carne, por cada peso invertido se está generando un $5.92 \%$ de remuneración, seguida por las medianas con $5.65 \%$ y en menor medida las pequeñas con 4.59 (Tabla 6); lo que resalta es que esta remuneración es muy parecida para los tres tipos de empresas, lo que puede señalar que la escala de la producción es importante solo en la magnitud de los resultados obtenidos, pero no tanto en el uso de los recursos, ya que los niveles de rentabilidad porcentual son similares.

\begin{tabular}{|l|r|r|r|}
\hline \multicolumn{1}{|c}{ Tamaño } & Pequeñas & \multicolumn{1}{c|}{ Medianas } & Grandes \\
\hline $\begin{array}{l}\text { Remuneración } \\
\text { absoluta }\end{array}$ & 17,353 & 60,276 & 184,935 \\
\hline $\begin{array}{l}\text { Costo total de } \\
\text { producción }\end{array}$ & 378,247 & $1,066,124$ & $3,126,506$ \\
\hline Relativa & $4.59 \%$ & $5.65 \%$ & $5.92 \%$ \\
\hline
\end{tabular}

Tabla 6 Remuneración al capital del productor por tipo de Unidad de producción. Precios reales 2019. IPP 2012=100 Fuente: Elaboración propia con datos de las U.P muestra de la S.P.R. de R.L.

Esta razón muestra la baja remuneración que se genera en las inversiones pecuarias que comparado con el nivel de riesgo que pueden sufrir, por la variabilidad de precios, enfermedades o otros fenómenos económicos o climáticos, representan un desestimulo a la inversión

\section{- Competitividad (RCP)}

La Relación de Costo privado (RCP) como medida de la competitividad, muestra que todas las unidades de producción son competitivas, al hacer un uso eficiente de los recursos, y el valor más pequeño refleja una mayor competitividad, Tabla 7.

De acuerdo con este indicador, las unidades grandes son consideradas las más competitivas, manejan los mayores volúmenes de producción y a pesar de tener deficiencias en su sistema de producción, la utilización de los recursos y el manejo adecuado de la producción, la hacen ser eficiente, aunque pueden plantearse mejoras significativas mediante capacitación y evaluación constante para determinar $\mathrm{y}$ monitorear el resultado de su sistema financiero. Para estas empresas, el costo de sus factores internos representa apenas un $40 \%$ del valor agregado generado en la producción de carne.

Le sigue en importancia las unidades pequeñas, lo que indica que estas empresas son competitivas a pesar del nivel de cabezas que manejan, el valor del RCP de 0.49 garantiza que la optimización y uso de recursos es adecuada, no obstante, es indispensable poner atención en el sistema de producción y hacer las modificaciones necesarias para ser más eficientes en el corral de engorda y generar una mayor cantidad de ingresos.

Para estas la unidades de producción con una Remuneración al Capital de $4.6 \%$ indica que una empresa rentable y competitiva y además explica el por qué continúan desarrollando su actividad, de ahí su explicación que puedan sobrevivir a pesar de lo errático que puedan ser los precios o los costos de producción que enfrenten en el mercado; sin embargo, cuando comparamos el valor de la inversión actual contra el valor de algunas inversiones existentes de bajo riesgo, podemos entender que financieramente, un porcentaje de excedentes por debajo del 5\%, no representa estímulos suficientes para seguir produciendo bajo el mismo esquema. 
Estas empresas reportan una menor ganancias en comparación con las otras unidades estudiadas; son pequeñas pero su estudio sirve para conocer el comportamiento del sistema de producción tradicional empleado en el estado de Morelos, y a su vez, evaluar la factibilidad de dicho sistema que por estadística es el más practicado por los ganaderos.

\begin{tabular}{|c|c|c|c|}
\hline Tamaño & Pequeñas & Medianas & Grandes \\
\hline $\begin{array}{ll}\text { Cost } & \text { Fact } \\
\text { Internos: } & \\
\end{array}$ & 16,598 & 62,703 & 121,283 \\
\hline Valor Agregado & 33,568 & 122,180 & 303,718 \\
\hline RCP & 0.49 & 0.51 & 0.40 \\
\hline
\end{tabular}

Tabla 7 Relación de Costo Privado por tipo de Unidad de producción. Precios reales 2019. IPP 2012=100

Fuente: Elaboración propia con datos de las U.P muestra de la S.P.R. de R.L.

Las unidades medianas también son competitivas aunque en menor proporción que las anteriores, sus resultados resultan interesante, puesto que son las que más inversiones realizan en cuanto a su infraestructura y calidad del ganado, a pesar de ser una unidad pequeña en desarrollo, el resultado de la Remuneración al Capital reportó un $5.7 \%$ indica que puede competir económicamente con las grandes unidades de producción. Al no reportar pérdidas monetarias $\mathrm{y}$ tener un equilibrio en su sistema de producción, es posible proponer una reestructura de gastos e inversión, que pueda modificar y corregir, las deficiencias observadas en su estado financiero.

\section{Conclusiones}

En la presente investigación se desarrolló un análisis de rentabilidad que fue evaluado bajo el esquema del Programa Federal "Proveedor Confiable" con la metodología de SENASICA. La aplicación del Programa "Proveedor Confiable" al sistema de producción de la S.P.R. de R.L. no presenta los resultados esperados, pues al no haber una cadena de comercialización que mejore el precio final del producto el productor no mejora sus ingresos por la producción de carne libre de Clembuterol.

El análisis de rentabilidad del sistema de producción implementado bajo el Programa y su impacto en el ingreso económico del productor se destaca lo siguiente:
- Todas las unidades de producción analizadas resultaron rentables, siendo las unidades grandes que por su tamaño $\mathrm{y}$ volumen de producción fueron las más rentables. Sin embargo, todas presentan una remuneración al capital que no supera el 5\% lo cual puede considerarse baja en relación con el riesgo que incurren en la producción.

En cuanto a los costos de producción son las unidades de producción grandes las que tienen un mayor nivel de inversión y en la compra de los insumos necesarios para la producción. El estudio muestra una comparación de costos detallada de cada uno de los sistemas de producción determinados como muestras, donde se puede entender que cuando existe un sistema de producción mal estructurado que no considera la aplicación de tecnología ni el conocimiento adecuado, la rentabilidad de la empresa puede verse afectada severamente. De igual forma se concluye que un alto costo de producción no necesariamente conduce a un alto nivel de ingresos.

- La producción de carne bajo los esquemas de producción libre de Clembuterol genera un valor agregado que ronda el $10 \%$ del valor del ingreso que cada tipo de unidad de producción genera, y otra vez, son las empresas grandes las que generan un mayor volumen de valor al interior del sector; por otra parte, en cuanto a la derrama económica que genera la actividad esta oscila alrededor del $90 \%$ del valor de los ingresos generados y son las unidades pequeñas las que representan el valor más grande de esta derrama.

- Finalmente, todas las empresas productoras de carne de ganado bovino libre de Clembuterol son competitivas, lo que muestra un uso eficiente del uso de sus recursos en la producción de carne, a pesar de los bajos niveles de precios que se reciben por la carne de buena calidad ya que no hay un mercado selectivo que indique que la producción se genera bajo los esquemas del programa "Proveedor Confiable", por lo que si se establecieran conjuntamente un esquema de comercialización que haga evidente esta forma de producción y se haga acreedora de un mejor nivel de precios. 
Algunas recomendaciones que pueden derivar se los resultados serían:

- En México se tiene la oportunidad de despuntar como país proveedor de carne de res de calidad y competitiva a nivel local y nacional, solo se necesita promover entre productores y consumidores las cualidades de la carne sana e inocua, además del desarrollo y la creación de nuevos nichos de mercado que puedan ser más redituables.

- El análisis de rentabilidad arrojó resultados donde se observa que el valor del ingreso depende de una serie de indicadores que por lo general el productor desconoce cuándo no es asesorado correctamente, además, los resultados muestran que la remuneración al capital no siempre es proporcional a la cantidad de bovinos producidos y mucho menos al tamaño de la U.P.

- A pesar de que las muestras no representan pérdidas económicas en la evaluación final, es importante mencionar que una remuneración al capital que no supera el $5 \%$, es considerada baja, pues se trata de una inversión de riesgo, por lo que sería recomendable establece políticas que fomenten la remuneración y así sea atractivo para los productores de carne de calidad.

\section{Referencias}

Aguilar Valdés, Alfredo (1997). Economía del agronegocio. México, Distrito Federal. Editorial Limusa 152 pág.

Cavallotti, V.B y Palacio, M.V.H. (2006). La ganadería Bovina en México y el Tratado de Libre Comercio. Reporte de investigación No. 37. México, CIESTAAM, Chapingo.

Gittinger, P. (1981). Análisis económico de proyectos agrícolas. España, Banco Mundial, Segunda edición, Madrid, 572 p.221

Guerrero. L.R y León, M.J.G. (2009). Elementos de la cadena productiva del sistema producto bovinos carne en México y sus repercusiones con la apertura comercial. UACH, México, Tesis de Licenciatura, Departamento de Zootecnia.
Guerra, E. Guillermo (1997). Economía del agronegocio. México, Distrito Federal. Editorial Limusa, 152p.

Huerta Suárez Carlos (2012). Tendencias en la ganadería: Visión actual, ponencia realizada en el Congreso Internacional de la Carne, celebrado el 6 de abril del año 2012, Ciudad de México.

J. Luna, Eduardo (2013). Trazabilidad: Término que llegó para quedarse en la producción animal. Grupo Inteligensa, México, pg. 68-102

López Ramírez, Daniel (2012). I Simposio de la carne de Morelos: Las expectativas del ganado de engorda y los productos cárnicos. Celebrado el 20 de octubre del 2012, Oaxtepec, Morelos, México.

Monke, E. y S. Pearson (1989). The Policy Analysis Matrix for agricultural development. Cornell University Press. Ithaca, New York. 279 p.

Monke, E. y S. Pearson (1987). The Policy Analysis Matrix a manual for practitioner. Office of policy Development and Program Review Bureau for Program and Policy Coordination U.S. Agency for International Development, Washington D. C. 221 p.

Morris, M. L. (1990). Determinación de la ventaja comparativa mediante el análisis del CRI: Pautas establecidas a partir de la experiencia del CIMMYT. Monografías en Economía del CIMMYT. No. 1. México D. F. CIMMYT.

Parkin, Michael (2004). Economía. México. Pearson Educación, Sexta edición, pg. 934.

Ronald, D. Kay (1981). Administración agrícola y ganadera; Planeación, control $e$ implementación. McGraw-Hill, Quinta impresión, Septiembre de 1987, 432 pg.

SAGARPA (2005). Manual de la administración de la empresa agrícola, México, Distrito Federal, Cuarta Edición, Volumen 4.

Secretaria de Economía (2012). Introducción al análisis de economía agrícola, México, Distrito Federal, Gobierno Federal, Primera Edición, 98 págs. 
Servicio Nacional de Sanidad, Inocuidad y Calidad Agroalimentaria. (2011). Programa Proveedor Confiable. Guía de operación y anexos - Proveedor Confiable (Libre de Clenbuterol).

Vizcarra (2013). El efecto de los costos de producción y la apertura de mercados locales. Ciudad de México. Editorial Trillas, 220 págs.

Zhigalin, M. M. (1985). Economía, organización y planificación de la producción agropecuaria. Editorial Progreso, URSS, 471p.

Zorrilla, R., José (2009). La engorda y el procesamiento de la carne mexicana de bovino. Guadalajara, México. Edtorial Limusa. 123 págs. 